\title{
Energy-Efficient Hybrid Precoding Scheme Based on Antenna Selection Technology in Massive Multiple-Input Multiple- Output Systems
}

\author{
Jian Jun Ding and Jing Jiang $\mathbb{D}$ \\ College of Communication and Information Engineering, Xi'an University of Posts and Telecommunications, Xi'an, Shanxi, China \\ Correspondence should be addressed to Jing Jiang; jiangjing@xupt.edu.cn
}

Received 31 August 2018; Accepted 4 March 2019; Published 2 May 2019

Guest Editor: Raed A. Abd-Alhameed

Copyright (C) 2019 Jian Jun Ding and Jing Jiang. This is an open access article distributed under the Creative Commons Attribution License, which permits unrestricted use, distribution, and reproduction in any medium, provided the original work is properly cited.

\begin{abstract}
Hybrid precoding is a promising technology for massive multiple-input multiple-output (MIMO) systems. It can reduce the number of radio frequency (RF) chains. However, the power consumption is still very high owing to the large-scale antenna array. In this paper, we propose an energy-efficient precoding scheme based on antenna selection technology. The precoding scheme greatly increases the energy efficiency (EE) of the system. In the first step, we derive an exact closed-form expression of EE. Meanwhile, we further study the relationship between the number of transmit antennas and EE on the basis of the exact closed-form expression of EE. We prove that there exists an optimal value. When the number of transmit antennas equals to the value, the EE of the system can reach the maximum by a proper hybrid precoding scheme. Then, we propose an antenna selection algorithm to select antennas from the transmit antennas. And the number of selected antennas equals to the optimal value. Subsequently, we design the analog precoder based on a codebook to maximize the equivalent channel gain. At last, we further improve the EE by baseband digital precoding. The precoding algorithm we proposed offers a compromise between spectral efficiency (SE) and EE in millimeter wave (mmWave) massive MIMO systems. Finally, simulation results validate our theoretical analysis and show that a substantial EE gain can be obtained over the precoding scheme we proposed without large performance loss.
\end{abstract}

\section{Introduction}

Millimeter wave band communication recently acquires more and more attention owing to its great advantages [1-4]. A beneficial feature of mmWave is that many antenna arrays can be packed into a small dimension owing to the small wavelength [5-11]. However, the problem is that the availability of multiantennas causes high interference between different users and high hardware complexity. Fortunately, precoding can eliminate interference between different users and reduce hardware complexity. Precoding is therefore more favored over mmWave MIMO communication systems. Simple linear precoding schemes, for example, zero forcing (ZF) and minimum mean square error precoding (MMSE), are virtually optimal. However, the digital processing in the MIMO system requires a dedicated base-band and radio frequency (RF) chain for every antenna element. Owing to the large amount of antennas deployed in mmWave massive MIMO systems, the costs are very huge and it is impossible to popularize in practice [12]. Researchers have widely studied on the reduction of the hardware cost. A hybrid precoding scheme comprising both digital and analog processing is capable of reducing the number of RF chains greatly. Therefore, the hybrid precoding scheme is widely used in mmWave MIMO systems. In consideration of a single-user scenario, a fully connected architecture-based hybrid precoding scheme is proposed in $[13,14]$, where each RF chain is connected to all antennas by analog phase shifters (APSs) and RF adders. The number of RF chains is greatly reduced in the fully connected architecture. However, in the fully connected architecture, the number of APSs is equal to the product of the number 
of RF chains and the number of antennas. The cost of hardware is still excessive owing to the large amount of antenna elements. Different from the fully connected architecture, a partially connected architecture-based hybrid precoding scheme is proposed in [15-19], where each RF chain only connected to an antenna subarray and the number of APSs equals to the number of antennas. Compared with the fully connected architecture, the number of phase converters and energy consumption is greatly reduced. In the partially connected precoding scheme, the mapping relationship between the antennas and RF chains is predetermined, but the channel condition is time-varying. Thus, the partially connected architecture cannot guarantee that the mapping relationship is optimal under different channel conditions. A dynamic subarray architecture-based hybrid precoding scheme is proposed in [20], where an adaptive antenna selection network is added between RF chains and antenna elements to enhance spectral efficiency (SE). All the previous work is aimed at improving the SE of the system. In the fully connected architecture-based and partially connected architecture-based architecture hybrid precoding schemes, all the transmitted antennas are activated. In this paper, we propose an energy-efficient precoding scheme with considerably reduced energy consumption and assume that not all transmitting antennas are activated. We jointly optimize the number of activated antennas, analog precoding matrix, and digital precoding matrix to maximize the $\mathbf{E E}$ of the system. Firstly, instead of designing the digital and analog precoder directly, we perform an antenna selection procedure before digital and analog precoding. Then, we further optimize the performance in terms of EE by digital and analog precoding.

The contributions existing in this paper are summarized as follows:

(1) An exact closed-form expression of $\mathbf{E E}$ was derived in this paper, and we derived an optimum number of antennas for maximizing the $\mathrm{EE}$ according to the exact closed-form expression of $\mathbf{E E}$

(2) In previous precoding schemes, all the antennas are activated. In this paper, not all the transmit antennas are activated (an antenna is activated, which means the antenna is used to transmit message). Based on channel state information and the exact closed-form expression of EE, we select antennas from the transmitting antennas to activate and the remaining antennas will be temporarily closed

(3) In the prior hybrid precoding scheme, we design the analog precoder and the digital precoder according to the channel state information $\mathbf{H}$ which was obtained by channel estimation to improve the sum rate and reduce the interference between different users. The hybrid precoding scheme we proposed differs from the prior hybrid precoding scheme. The transmitter first performs an antenna selection process according to the channel state information and then optimizes system performance in terms of EE by analog and digital precoding. Through the scheme, the number of antennas and power consumption is reduced, and the $\mathbf{E E}$ is improved

(4) An energy-efficient hybrid precoding scheme for a single user in mmWave systems is developed in this paper. First, we calculate the optimal number of transmit antennas and then the antenna selection algorithm is used to select the subset of transmit antennas. Then, we use the analog precoding scheme to maximize the gain of the equivalent channel between BS and objective users. Furthermore, we use the digital precoding scheme to maximize the $\mathbf{E}$ $\mathbf{E}$ of the system

The rest of this paper is organized as follows: Section 2, Section 3, and Section 4 introduce the system model, channel model, and power consumption model of the system, respectively. In Section 5, we introduce the EE optimization problem and propose an antenna selection algorithm. In Section 6 , we proposed a hybrid beamforming algorithm. Computer simulation results are shown in Section 7. Finally, conclusions are drawn in Section 8.

Notations: we use the following notation throughout the paper. A denotes a matrix; $\mathbf{a}$ is a vector; $\alpha$ denotes a scalar; $\mathbf{A}^{\mathrm{T}}$ denotes the transpose of $\mathbf{A} \cdot \mathbf{A}^{\mathbf{H}}$ denotes the conjugate of $\mathbf{A}$. $\mathbf{A}^{-1}$ denotes the inversion of $\mathbf{A} . \mathbf{A}(i, j)$ denotes the $(i, j)$-th element of $\mathbf{A}$. We express $\|\mathbf{A}\|_{F}^{2}$ as $\operatorname{Tr}\left(\mathbf{A} \mathbf{A}^{\mathbf{H}}\right)$. $\mathbf{I}$ is an identity matrix. The acronym s.t. denotes "subject to," and i.i.d. denotes "independent and identically distributed." $\mathbb{E}(\mathbf{A})$ denotes the mathematical expectation of $\mathbf{A}$, and $\mathscr{C} \mathcal{N}$ denotes complex Gaussian distribution.

\section{System Model}

In this paper, we consider a downlink SU-MIMO mmWave system where a base station (BS) is equipped with $N_{\mathrm{t}}$ antennas and $N_{\mathrm{RF}}^{\mathrm{t}} \mathrm{RF}$ chains to serve a single user which has $N_{\mathrm{RF}}^{\mathrm{r}}$ $\mathrm{RF}$ chains and $N_{\mathrm{r}}$ antennas. The number of RF chains satisfies $N_{\mathrm{s}} \leq N_{\mathrm{RF}}^{\mathrm{t}} \leq N_{\mathrm{t}}$ to guarantee multistream transmission.

First, $N_{\mathrm{s}}$ transmit data streams at the BS are processed by a digital precoder $F_{B B}$ in the baseband and then processed by an analog precoder $\mathrm{F}_{\mathrm{RF}}$ (RF precoder using analog circuitry). $\mathbf{F}_{\mathrm{RF}}$ of size $N_{\mathrm{t}} \times N_{\mathrm{RF}}^{\mathrm{t}}$ denotes the transmitting analog beam former. $\mathrm{F}_{\mathrm{BB}}$ denotes the base band digital precoder satisfying $\left\|\mathbf{F}_{\mathbf{R F}} \mathbf{F}_{\mathbf{B B}}\right\|_{F}^{2}=\rho$, and $\rho$ denotes the total transmit power. Notably, the RF precoder can realize only phase changes because of phase-only control. For hybrid precoding systems as shown in Figure 1, the received signal vector of the objective user can be expressed by

$$
\mathbf{y}=\mathrm{HF}_{\mathrm{RF}} \mathrm{F}_{\mathrm{BB}} s+\mathbf{n},
$$

where $\mathbf{y} \in C^{N_{\mathrm{r}} \times 1}$ denotes the received signal vector. $\mathbf{H}=$ $\left[h_{1}, h_{2}, \cdots, h_{N_{\mathrm{r}}}\right]^{T}$ denotes the $N_{\mathrm{r}} \times N_{\mathrm{t}}$ channel matrix with $h_{k}$ denoting the channel vector between the BS and the $k$ -th receiving antenna. $s$ denotes the transmitted signal vector, satisfying $\mathrm{E}\left(\mathbf{s s}^{\mathbf{H}}\right)=\mathbf{I}_{N_{\mathrm{r}}}$. And $N_{\mathrm{s}}$ represents the number of data streams. $\mathbf{n}$ is the vector of additive complex 


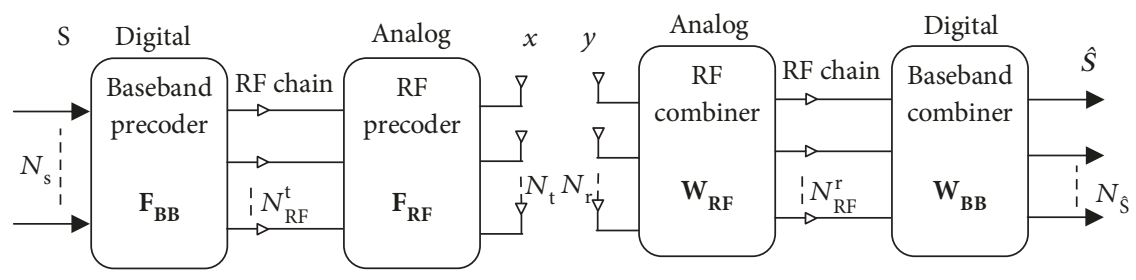

Figure 1: A mmWave single-user MIMO system.

Gaussian noise with zero mean and variance $\sigma^{2} . \sigma^{2}$ denotes the power of noise.

\section{Channel Model}

Since mmWave channels have high free-space path loss, the mmWave propagation can be perfectly characterized by a clustered channel model [21] in this paper. The SalehValenzuela model was used in this paper.

$$
\mathbf{H}=\sqrt{\frac{N_{\mathrm{r}} N_{\mathrm{t}}}{N_{\mathrm{cl}} N_{\text {ray }}}} \sum_{i=1}^{N_{\mathrm{cl}}} \sum_{l=1}^{N_{\text {ray }}} a_{i l} a_{\mathrm{r}}\left(\phi_{i l}^{\mathrm{r}}, \theta_{i l}^{\mathrm{r}}\right) \alpha_{\mathrm{t}}\left(\phi_{i l}^{\mathrm{t}}, \theta_{i l}^{\mathrm{t}}\right)^{H},
$$

$$
\alpha\left(\varphi_{i l}, \theta_{i l}\right)=\frac{1}{\sqrt{M}}\left[1, \cdots e^{j(2 \pi / \lambda) \mathrm{d}\left(p \sin \varphi_{i l} \theta_{i l}+q \cos \theta_{i l}\right)}, \ldots\right.
$$

$d$ denotes the distance between antennas, and $\lambda$ denotes the wavelength of the signal. $p$ satisfies $0 \leq p \leq \sqrt{M}$, the same with $q$. $p$ and $q$ are the antenna indices in the $2 \mathrm{D}$ plane.

\section{Power Consumption Model}

This section discusses the power consumption model of the downlink single-user MIMO mmWave system. The power consumption model of the system [22-24] can be denoted by

$$
P=\rho P_{\mathrm{t}}+P_{\mathrm{c}},
$$

where $\rho$ denotes the inefficiency factor of the power amplifier (PA). $P_{\mathrm{t}}$ denotes the power consumption of the transmitter, and $P_{\mathrm{t}}=\left\|\mathbf{F}_{\mathrm{RF}} \mathrm{F}_{\mathrm{BB}}\right\|_{F}^{2} . \quad P_{\mathrm{c}}$ consists of the dynamic power consumption $P_{\text {dyn }}$ and static power consumption $P_{\text {stac }}$. $P_{\text {dyn }}$ can be denoted by

$$
P_{\text {dyn }}=N_{\mathrm{RF}}^{\mathrm{t}} P_{\mathrm{RF}}+N_{\mathrm{RF}}^{\mathrm{t}} N_{\mathrm{t}} P_{\mathrm{s}}
$$

where $N_{\mathrm{RF}}^{\mathrm{t}} P_{\mathrm{RF}}$ denotes the power consumption of all RF chains. The number of APSs is $N_{\mathrm{RF}}^{\mathrm{t}} N_{\mathrm{t}}$; thus, the power consumption of phase shifters is $N_{\mathrm{RF}}^{\mathrm{t}} N_{\mathrm{t}} P_{\mathrm{s}}$, where $P_{\mathrm{s}}$ where the number of cluster and the number of rays can be expressed by $N_{\mathrm{cl}}$ and $N_{\text {ray }}$, and the gain of the $i$-th ray in the $j$-th cluster can be expressed as $\alpha_{i j}$. We assumed that $\alpha_{i j}$ satisfies the complex Gaussian distribution $\mathscr{C} \mathscr{N}\left(0, \sigma_{\alpha, i}^{2}\right)$ and $\sigma_{\alpha, i}^{2}$ satisfies $\sum_{j=1}^{N_{\mathrm{cl}}} \sigma_{\alpha, i}^{2}=\widehat{\beta}$, and $\widehat{\beta}$ is a normalization factor, where $\alpha\left(\phi_{i l}^{\mathrm{r}}, \theta_{i l}^{\mathrm{r}}\right)$ and $\alpha\left(\phi_{i l}^{\mathrm{t}}, \theta_{i l}^{\mathrm{t}}\right)$ denotes the receive and transmit array response vectors, respectively. $\phi_{i l}^{\mathrm{r}}\left(\phi_{i l}^{\mathrm{t}}\right)$ and $\theta_{i l}^{\mathrm{r}}\left(\theta_{i l}^{\mathrm{t}}\right)$ denote the azimuth and elevation angles of arrival and departure, respectively.

In this paper, we adopt a uniform square planar array with $\sqrt{M} \times \sqrt{M}$ antenna elements.

$\cdots, e^{\left.j(2 \pi / \lambda)((\sqrt{M}-1)) \mathrm{d} \sin \varphi_{i l} \sin \theta_{i l}+((\sqrt{M}-1)) \cos \theta_{i l}\right]^{1 / 2} .}$

denotes the energy consumption of APS and $P_{\mathrm{RF}}$ denotes the power consumption of the RF chain.

\section{Problem Formulation and Antenna Selection Algorithm}

5.1. Problem Formulation. First, we consider the relationship between system capacity and signal-to-noise ratio. According to Shannon's theorem, the relationship between system capacity and SNR can be expressed by the following formula:

$$
\begin{aligned}
C & =\log _{2} \operatorname{det}(\mathbf{I}+\mathrm{SNR}) \\
& =\log _{2} \operatorname{det}\left(\mathbf{I}+\frac{\left(\mathbf{H F}_{\mathbf{R F}} \mathbf{F}_{\mathbf{B B}}\right)^{*}\left(\mathbf{H F}_{\mathbf{R F}} \mathbf{F}_{\mathbf{B B}}\right)}{\sigma^{2}}\right) .
\end{aligned}
$$

Once the antenna set is determined, the corresponding channel matrix is determined. We can define the channel matrix as $\mathbf{H}_{\text {sel }}^{\mathrm{t}}$. Equation (6) can be denoted as

$$
C\left(S_{\text {sel }}^{\mathrm{t}}\right)=\log _{2} \operatorname{det}\left(\mathbf{I}+\frac{\left(\mathbf{H}_{\mathrm{sel}}^{\mathrm{t}} \mathbf{F}_{\mathrm{RF}} \mathbf{F}_{\mathbf{B B}}\right)^{*}\left(\mathbf{H}_{\mathrm{sel}}^{\mathrm{t}} \mathbf{F}_{\mathrm{RF}} \mathbf{F}_{\mathbf{B B}}\right)}{\sigma^{2}}\right) .
$$


The objective of this paper is to select the transmit antenna subset according to channel state information and design the hybrid precoders to maximize the $\mathbf{E E}$ of the system. The problem can be formulated as

$$
\begin{aligned}
\left\{\mathbf{F}_{\mathbf{R F}}, \mathbf{F}_{\mathbf{B B}}, S_{\text {sel }}^{t}\right\}=\underset{\mathbf{F}_{\mathrm{RF}}, \mathbf{F}_{\mathbf{B B}}, S_{\text {sel }}^{\mathrm{t}}}{\arg \max }\left(\frac{\log \left|\mathbf{I}+\left(1 / \sigma_{n}^{2}\right)\left\|\mathbf{H}\left(S_{\text {sel }}^{\mathrm{t}}\right) \mathbf{F}_{\mathbf{R F}} \mathbf{F}_{\mathbf{B B}}\right\|_{F}^{2}\right|}{P_{\mathrm{c}}+\left\|\mathbf{F}_{\mathbf{R F}} \mathbf{F}_{\mathbf{B B}}\right\|_{F}^{2}}\right) \\
\text {. s.t. } \quad\left\|\mathbf{F}_{\mathbf{R F}} \mathbf{F}_{\mathbf{B B}}\right\|_{F}^{2} \leq P_{\text {tot }},
\end{aligned}
$$

where $S_{\text {sel }}^{t}$ denotes the selected antenna subset. The elements of $S_{\text {sel }}^{t}$ are the index of selected antennas (e.g., $S_{\text {sel }}^{t}$ $=\{1,3,4\}$ denotes that the selected antennas are the first, second, and fourth antennas of the BS). It is worth pointing out that the constraint in equation (8) is nonconvex, because of the coupling between the analog precoding matrix and digital precoding matrix. This makes equation (8) to be a difficult problem to solve. Fortunately, we can decouple the optimization problem into two convex optimization problems. First, we focus on the antenna selection process. We select the optimal subset of the antennas from the transmit antennas according to channel matrix $\mathbf{H}$. Then, with the channel matrix between the selected antenna and objective user, the hybrid precoding scheme can be adopted to improve the EE of the system. Specially, we adopt the analog precoding to maximize the gain of the equivalent channel and digital precoding to maximize the EE.

At first, we would briefly introduce the $\mathbf{E E}$ of the system without consideration of hybrid precoding. The EE is defined as the throughput divided by the total energy consumed, which can be denoted by

$$
\mathrm{EE}=\frac{\mathbb{E}(\mathbf{I})}{P_{\text {tot }}}=\frac{\mathbb{E}(\mathbf{I})}{P_{\mathrm{c}}+P_{\mathrm{t}}},
$$

where $\mathbb{E}(\mathbf{A})$ denotes the mathematical expectation of $\mathbf{A}$, and I denotes the mutual information. If the power consumption of the receiver is taken into account, the circuit power consumption model can be expressed:

$$
\begin{aligned}
P_{\mathrm{c}} \approx & N_{\mathrm{t}}\left(P_{\mathrm{dac}}+P_{\text {mixer }}+P_{\text {filt }}\right)+2 P_{\text {syn }} \\
& +N_{\mathrm{r}}\left(P_{\ln a}+P_{\text {mixer }}+P_{\text {ifa }}+P_{\text {adc }}\right),
\end{aligned}
$$

where $N_{\mathrm{t}}$ and $N_{\mathrm{r}}$ denote the number of transmitters and receivers, respectively, and $P_{\text {dac }}, P_{\text {mixer }}, P_{\text {filt }}, P_{\text {syn }}, P_{\text {lna }}, P_{\text {ifa }}$, and $P_{\text {adc }}$ are the power consumption of the digital analog converter, the mixer, the filter at the transmit side, the synthesizer, the low-noise amplifier, the intermediatefrequency amplifier, the filters at the receiver side, and the analog digital converter, respectively. We set

$$
\begin{aligned}
& P_{1}=2 P_{\text {syn }}+N_{\mathrm{r}}\left(P_{\text {lna }}+P_{\text {mixer }}+P_{\text {ifa }}+P_{\text {adc }}\right)+P_{\mathrm{t}}, \\
& P_{2}=\left(P_{\ln a}+P_{\text {mixer }}+P_{\text {ifa }}+P_{\text {adc }}\right), \\
& \mathbf{E E}=\frac{\mathbb{E}(\mathbf{I})}{P_{1}+N_{\mathrm{t}} P_{2}},
\end{aligned}
$$

where $P_{2}$ is a very small value much less than one.

Lemma 1. Suppose there exists a communication system. $N_{\mathrm{t}}$ transmit antennas were equipped by the BS, and $N_{\text {sd }}$ antennas are selected from these transmitter antennas; the distribution of mutual information is approximately [25] given by

$$
\begin{array}{r}
I_{\text {sel }} \sim \mathscr{F} \mathcal{N}\left(\log _{2}\left(1+\left(1+\ln \frac{N_{\mathrm{t}}}{N_{\text {sel }}}\right) \rho N_{\text {sel }}\right)\right. \\
\left.\cdot \frac{\left(\log _{2} e\right)^{2} \rho^{2} N_{\text {sel }}\left(2-\left(N_{\text {sel }} / N_{\mathrm{t}}\right)\right)}{\left(1+\left(1+\ln \left(N_{\mathrm{t}} / N_{\text {sel }}\right)\right) \rho N_{\text {sel }}\right)}\right)
\end{array}
$$

Thus, $\mathbb{E}\left(I_{\text {sel }}\right)=\log _{2}\left(1+\left(1+\ln \left(N_{\mathrm{t}} / N_{\text {sel }}\right)\right) \rho N\right)$. So, the closed-form expression of the EE becomes

$$
\mathbf{E E}=\frac{\mathbb{E}\left(\mathbf{I}_{\text {sel }}\right)}{P_{\text {tot }}} \approx \frac{\log _{2}\left[1+\left(1+\ln \left(N_{\mathrm{t}} / N_{\text {sel }}\right)\right) \rho N_{\text {sel }}\right]}{\rho+P_{1}+N_{\text {sel }} P_{2}} .
$$

Next, we will analyze the effects of the number of transmitted antennas on the EE.

Lemma 2. If the power consumption of the mmWave MIMO system can be modeled as the addition of the transmit power $P_{\mathrm{t}}$ and the circuit power consumption $P_{\mathrm{c}}$, EE increases at first and then decreases as the number of selected transmit antennas $N_{\text {sel }}^{\mathrm{t}}$ increases.

Proof 1. EE can be expressed as a function of $N_{\text {sel }}$ :

$$
\eta\left(N_{\text {sel }}\right)=\frac{\log _{2}\left[1+\left(1+\ln \left(N_{\mathrm{t}} / N_{\text {sel }}\right)\right) \rho N_{\text {sel }}\right]}{\rho+P_{1}+N_{\text {sel }} P_{2}} .
$$

We can take the first derivative of $\eta\left(N_{\text {sel }}\right)$

$$
\begin{aligned}
\frac{\partial\left(\eta\left(N_{\text {sel }}\right)\right)}{\partial N_{\text {sel }}} & =\frac{\left(\log _{2}\left(1+\left(1+\ln \left(N_{\mathrm{t}} / N_{\text {sel }}\right)\right) \rho N_{\text {sel }}\right)\right)^{\prime}\left(\rho+P_{1}+N_{\text {sel }} P_{2}\right)}{\left(\rho+P_{1}+N_{\text {sel }} P_{2}\right)^{2}}+\frac{\left(\log _{2}\left(1+\left(1+\ln \left(N_{\mathrm{t}} / N_{\text {sel }}\right)\right) \rho N_{\text {sel }}\right)\right)^{\prime} P_{2}}{\left(\rho+P_{1}+N_{\text {sel }} P_{2}\right)^{2}} \\
& =\frac{\left(\rho+P_{1}+N_{\text {sel }} P_{2}\right) \rho \ln \left(N_{\mathrm{t}} / N_{\text {sel }}\right)}{q\left(N_{\text {sel }}\right)}-\frac{P_{2}\left[1+\left(1+\ln \left(N_{\mathrm{t}} / N_{\text {sel }}\right)\right) \rho N_{\text {sel }}\right] \ln \left[1+\left(1+\ln \left(N_{\mathrm{t}} / N_{\text {sel }}\right)\right) \rho N_{\text {sel }}\right]}{q\left(N_{\text {sel }}\right)},
\end{aligned}
$$


setting $q\left(N_{\text {sel }}\right)=\left(\rho+P_{1}+N_{\text {sel }} P_{2}\right)^{2}$. Because the denominator of (17) is positive, the denominator of (17) has no effect on the monotonicity of function (17). Setting the numerator of (17) as $p\left(N_{\text {sel }}\right)$,

$$
\begin{aligned}
p\left(N_{\text {sel }}\right)= & \left(\rho+P_{1}+N_{\text {sel }} P_{2}\right) \rho \ln \frac{N_{\mathrm{t}}}{N_{\text {sel }}} \\
& -P_{2}\left[1+\left(1+\ln \frac{N_{\mathrm{t}}}{N_{\text {sel }}}\right) \rho N_{\text {sel }}\right] \\
& * \ln \left(1+\left(1+\ln \frac{N_{\text {tl }}}{N_{\text {sel }}}\right) \rho N_{\text {sel }}\right) .
\end{aligned}
$$

Then, we take the first derivative of $p\left(N_{\text {sel }}\right)$ again:

$$
\begin{aligned}
p^{\prime}\left(N_{\text {sel }}\right)= & -\frac{\left(\rho+P_{1}+N_{\text {sel }} P_{2}\right)}{N_{\text {sel }}} \\
& -P_{2} \rho \ln \left[1+\left(\ln \frac{N_{\mathrm{t}}}{N_{\text {sel }}}+1\right) \rho N_{\text {sel }}\right] .
\end{aligned}
$$

It is obvious that $p^{\prime}\left(N_{\text {sel }}\right)$ is a negative value. So, $p\left(N_{\text {sel }}\right)$ is a decreasing function of $\left(N_{\text {sel }}\right)$; thus, $p\left(N_{\mathrm{t}}\right) \leq p\left(N_{\text {sel }}\right) \leq p(1)$ :

$$
p\left(N_{\mathrm{t}}\right)=-P_{2}\left(1+N_{\mathrm{t}} \rho\right)<0,
$$

and

$$
\begin{aligned}
p(1)= & \left(\rho+P_{1}+P_{2}\right) \rho \ln N_{\mathrm{t}}-\cdots--P_{2}\left[1+\left(1+\ln \left(N_{\mathrm{t}}\right)\right) \rho\right] \\
& \cdot \ln \left[1+\left(1+\ln N_{\mathrm{t}}\right) \rho\right] .
\end{aligned}
$$

We do not know whether $p(1)$ is a positive or negative value. However, equation (21) can also be viewed as a function of $\rho$. We set $P(1)=f(\rho)$. Then, we take the first derivative of $\rho$.

$$
\begin{aligned}
f^{\prime}(\rho)= & \left(2 \rho+P_{1}+P_{2}\right) \ln \left(N_{\mathrm{t}}\right)-\cdots-P_{2}\left(\left[\left(1+\ln N_{\mathrm{t}}\right)\right]\right. \\
& \left.\cdot \ln \left[\left(1+\ln \left(N_{\mathrm{t}}\right)\right) \rho\right]\right)-\cdots--\left(P_{2}\left(1+\ln \left(N_{\mathrm{t}}\right) \rho\right)\right. \\
& \left.\cdot\left(\frac{1+\ln \left(N_{\mathrm{t}}\right)}{1+\ln \left(N_{\mathrm{t}}\right)}\right)\right) .
\end{aligned}
$$

We take the first derivative of (22) again in respective of $\rho$, we can conclude that

$$
f^{\prime \prime}(\rho) \geq 2 \ln \left(N_{\mathrm{t}}\right)-P_{2}\left(1+\ln \left(N_{\mathrm{t}}\right)\right)
$$

As mentioned earlier in this paper, $P_{2}$ is a very small number much less than one, so $f^{\prime \prime}(\rho)>0$. Then, $f^{\prime}(\rho)>$ $f^{\prime}(0)>0$, so $f(\rho)$ is an increasing function of $\rho$. Besides,

$$
\lim _{\rho \rightarrow 0} f(\rho)=0,
$$

so $P(1)=f(\rho)>0$. So, $\eta$ increases first and then decreases with the increase in $N_{\text {sel }}$. Assume that $N_{0}$ satisfies $p\left(N_{0}\right)=0$. With mathematical knowledge, we can conclude that

(1) if $N_{\text {sel }} \in\left[1, N_{0}\right]$, the $\mathbf{E E}$ of the system increases with the increase in $N_{\text {sel }}$

(2) if $N_{\text {sel }} \in\left[N_{0},+\infty\right)$, the $\mathbf{E E}$ of the system decreases with the increase in $N_{\text {sel }}$

That is to say, there exists an optimal number of antenna $N_{\text {sel }}^{\text {opt }}$ that can maximize the $\mathbf{E E}$ of the system.

5.1.1. Discussion. Based on the system model and power consumption model that we proposed, EE increases at first and then decreases with the increase in the number of antennas. This is because with the increase in the number of selected antennas, the power consumption increases linearly. However, this is not true for the sum rate of the system. When the number of antennas increases to a certain value, the increase in the sum rate is not enough to compensate for the increase in the energy consumption of the system, so the $\mathbf{E E}$ of the system will reduce.

Denote the selected transmit antenna subset as $S_{\text {sel }}^{t}$, the number of active transmits $L_{\mathrm{t}}$ is $\left|S_{\text {sel }}^{\mathrm{t}}\right|$ where $|A|$ denotes the cardinality of $A$. EE could also be also expressed as

$$
\mathbf{E E}=\frac{\log _{2} \operatorname{det}\left(I+\left(\left\|\mathbf{H}\left(\mathbf{S}_{\text {sel }}^{\mathrm{t}}\right) \mathbf{F}_{\mathrm{RF}} \mathbf{F}_{\mathrm{BB}}\right\|_{F}^{2} / \sigma_{n}^{2}\right)\right)}{P_{\mathrm{c}}+\rho P_{\mathrm{t}}}(\text { bit/Hz/joule }),
$$

where $\mathbf{H}\left(\mathbf{S}_{\text {sel }}^{\mathrm{t}}\right)$ is the channel matrix of size $N_{\mathrm{r}} \times N_{\text {sel }}$ between the $L_{\mathrm{t}}$ transmit antenna and the $N_{\mathrm{r}}$ receive antennas. The objective of antenna selection can be expressed as

$$
\left\{S_{\text {sel }}^{t}, \mathbf{F}_{\mathrm{RF}}, \mathbf{F}_{\mathrm{BB}}\right\}=\underset{S_{\text {sel }}^{t}, \mathbf{F}_{\mathrm{RF}} \mathbf{F}_{\mathrm{BB}}}{\arg \max } \operatorname{EE}\left(\mathbf{S}_{\mathrm{sel}}^{\mathrm{t}}, \mathbf{F}_{\mathrm{RF}}, \mathbf{F}_{\mathrm{BB}}\right) \quad \text { s.t. } \quad \mathrm{SNR}>\gamma,
$$

where (26) is a nonconvex optimization problem [26-29]. It is often intractable to directly solve, because it is a joint optimization problem over three variables. We propose a suboptimal algorithm to decouple the nonconvex optimization problem into a convex optimization problem that can be solved directly. First, just as it is proved above, there exists an optimal value $N_{\text {opt }}$. When the number of transmitter antennas equals to the value, the $\mathbf{E E}$ of the system can achieve an optimal value. Thus, the proposed algorithm will be divided into three parts.

At first, as we know, there exists an optimal value $N_{\text {opt }}$ that maximizes the EE. Thus, we can select $N_{\text {opt }}$ antennas from $N_{\mathrm{t}}$ transmit antennas to optimize the EE of the system before hybrid precoding.

Then, an analog precoder is designed to maximize the gains of the equivalent channel, where the equivalent channel $\mathbf{H}_{\text {eff }}$ is defined as $\mathbf{H}_{\mathrm{sel}} \mathbf{F}_{\mathrm{RF}}$. At this step, it is assumed that the digital precoding matrix $\mathbf{F}_{\mathbf{b b}}$ is fixed. 
The design of digital precoder will become an easily solved optimization problem with a single variable after the analog precoding. The digital precoding matrix will be designed based on the maximized EE criterion.

The proposed antenna select algorithm and hybrid precoding scheme will be discussed in the next section.

5.2. Antenna Selection Algorithm. In this section, a lowcomplexity antenna select algorithm is proposed which enables the BS to reduce the number of transmit antennas and without large performance loss. The antenna selection algorithm is described as below.

As discussed above, EE increases first and then decreases with the increase in transmit antenna in mmWave massive MIMO systems. So, we can select $N_{\text {opt }}$ antennas from $N_{\mathrm{t}}$ transmit antennas to optimize the $\mathbf{E E}$ of the system before hybrid precoding at first.

The pseudocode of the proposed antenna selection algorithm is summarized in Algorithm 1, which can be explained as follows. At the beginning, we formulate the EE of the system as the sum rate $R$ divided by energy consumption $P_{\mathrm{c}}$ and set the EE denoted in (13). During the antenna selection, in step 1, step 2, and step 3, we calculate the EE of the mmWave MIMO system with different numbers of antennas. In step 4, we find the optimal number of antennas $N_{\text {opt }}$ by comparing EE under different conditions. Then, in step 6, step 7, and step 8, we choose $N_{\text {opt }}$ optimal antennas from $N_{\mathrm{t}}$ transmitted antennas by comparing the Frobenius norm of the channel vector of each antenna. Finally, the $N_{\text {opt }}$ antennas are used to transmit information. Note that the antenna selection algorithm enables the system to adaptively choose the transmit antenna under different channel conditions to maximize the EE of the system.

\section{The Design of the Hybrid Precoder}

In this section, we will discuss the design of the hybrid precoder for the mmWave massive MIMO system under the condition that the transmit antenna subset $S_{\text {sel }}^{t}$ has been obtained.

As the antenna selection process effectively reduces the number of transmit antennas, the power consumption of the system is reduced. After the antenna subset has been obtained, analog precoding and base band digital precoding will be utilized to improve the EE of the system. The optimization problem (26) can be written as

$$
\left\{\mathbf{F}_{\mathbf{R F}}, \mathbf{F}_{\mathbf{B B}}\right\}=\underset{\mathbf{F}_{\mathrm{RF}}, \mathbf{F}_{\mathrm{BB}}}{\arg \max }\left(\frac{\mathbf{R}\left(\mathbf{F}_{\mathbf{R F}} \mathbf{F}_{\mathbf{B B}}\right)}{\mathbf{P}\left(\mathbf{F}_{\mathbf{R F}} \mathbf{F}_{\mathbf{B B}}\right)}\right) \quad \text { s.t. } \quad\left\|\mathbf{F}_{\mathbf{R F}} \mathbf{F}_{\mathbf{B B}}\right\|_{F}^{2} \leq P_{\text {tot }} \text {, }
$$

where $R\left(\mathbf{F}_{\mathbf{R F}}, \mathbf{F}_{\mathbf{B B}}\right)$ denotes the sum rate of the proposed system and $P\left(\mathbf{F}_{\mathbf{R F}}, \mathbf{F}_{\mathbf{B B}}\right)$ denotes the energy consumption of the whole system.

$$
\begin{gathered}
R\left(\mathbf{F}_{\mathbf{R F}}, \mathbf{F}_{\mathbf{B B}}\right)=\log \left|\mathbf{I}_{\mathrm{N}_{\mathrm{r}}}+\mathbf{H}_{\text {sel }}^{\mathbf{t}} \mathbf{F}_{\mathbf{R F}} \mathbf{F}_{\mathbf{B B}} \mathbf{F}_{\mathbf{B B}}^{\mathbf{H}} \mathbf{F}_{\mathbf{R F}}^{\mathbf{H}}\left(\mathbf{H}_{\text {sel }}^{\mathbf{t}}\right)\right|, \\
P\left(\mathbf{F}_{\mathbf{R F}}, \mathbf{F}_{\mathbf{B B}}\right)=P_{\mathrm{c}}+\left\|\mathbf{F}_{\mathbf{R F}} \mathbf{F}_{\mathbf{B B}}\right\|_{F}^{2} .
\end{gathered}
$$

At first, an analog precoding algorithm is proposed to maximize the power of the received signal for the objective user [30-32]. Further, a digital precoder will be designed to maximize the EE of the system. However, it is difficult to solve problem (27) because of the coupling between two matrix variables: $\mathbf{F}_{\mathbf{R F}}$ and $\mathbf{F}_{\mathbf{B B}}$. We solve this problem by decoupling the joint optimization problem into two convex optimization problems.

Firstly, an analog precoder is designed that is aimed at maximizing the equivalent channel gain and assuming that the base-band precoding matrix $\mathbf{F}_{\mathbf{B B}}$ is fixed at this stage.

After the analog precoding matrix is obtained, the original optimization problem (27) becomes an easily solved convex optimization problem with one matrix variable $\mathbf{F}_{\mathbf{B B}}$, which can be expressed as

$$
\left\{\mathbf{F}_{\mathrm{BB}}\right\}=\underset{\mathbf{F}_{\mathrm{BB}}}{\arg \max } \mathbf{E E}\left(\mathbf{F}_{\mathrm{BB}}\right) \quad \text { s.t. } \quad\left\|\mathbf{F}_{\mathbf{R F}} \mathbf{F}_{\mathbf{B B}}\right\|_{F}^{2} \leq P_{\text {tot }} .
$$

Then, the optimal baseband precoding matrix $\mathbf{F}_{\mathbf{B B}}$ can be obtained by solving (29).

6.1. The Design of the Analog Precoder. In this section, we will discuss the optimal analog precoding matrix $\mathbf{F}_{\mathbf{R F}}$, where $\mathbf{F}_{\mathbf{R F}}=\left(f_{1}, f_{2}, \cdots f_{N_{\mathrm{rf}}}\right)$; the equivalent channel $\mathbf{H}_{\text {eff }}$ is defined as $\mathbf{H F}_{\mathbf{R F}}$. We rewrite the receive signal of the objective user

$$
\mathbf{y}=\mathbf{H}_{\mathrm{eff}} \mathrm{F}_{\mathrm{BB}} \mathrm{s}+\mathbf{n}
$$

The gain of the equivalent channel can be expressed as

$$
G\left(\mathbf{H}_{\text {eff }}\right)=\left\|\mathbf{H F}_{\mathbf{R F}}\right\|_{F}^{2} .
$$

We aim to find an analog precoding matrix $\mathbf{F}_{\mathbf{R F}}$ to maximize the equivalent channel gain based on codebook $\mathscr{F}$. $\mathscr{F}$ is a predefined RF beamforming codebook. $\mathscr{F}$ can be specified as a quantized matrix $\alpha_{T}\left(2 \pi i_{\varphi} / M_{\varphi}, 2 \pi j_{\theta} / M_{\theta}\right)$. For $i_{\varphi}$, it takes the value $0,1,2,3, \ldots, M_{\varphi}-1$, and for $j_{\theta}$, it takes the value $0,1,2,3, \ldots, M_{\theta}-1 . M_{\varphi}$ and $M_{\theta}$ denote the quantized precision of azimuth and elevation angles, respectively. The most optimal precoding matrix $\mathbf{F}_{\mathbf{R F}}$ is designed based on the following metric.

$$
\mathbf{f}_{i}=\underset{\mathbf{f}_{i} \in F}{\arg \max }\left\|\mathbf{H f}_{i}\right\|_{F}^{2}, \quad i=1,2,3, \cdots, N_{\mathrm{rf}} .
$$

The pseudocode of the proposed analog precoding algorithm is summarized in Algorithm 2.

At the beginning, we set $i=1$, and we search in the codebook $\mathscr{F}$ to find out an optimal vector $f_{i} \in \mathscr{F}$ which has the 


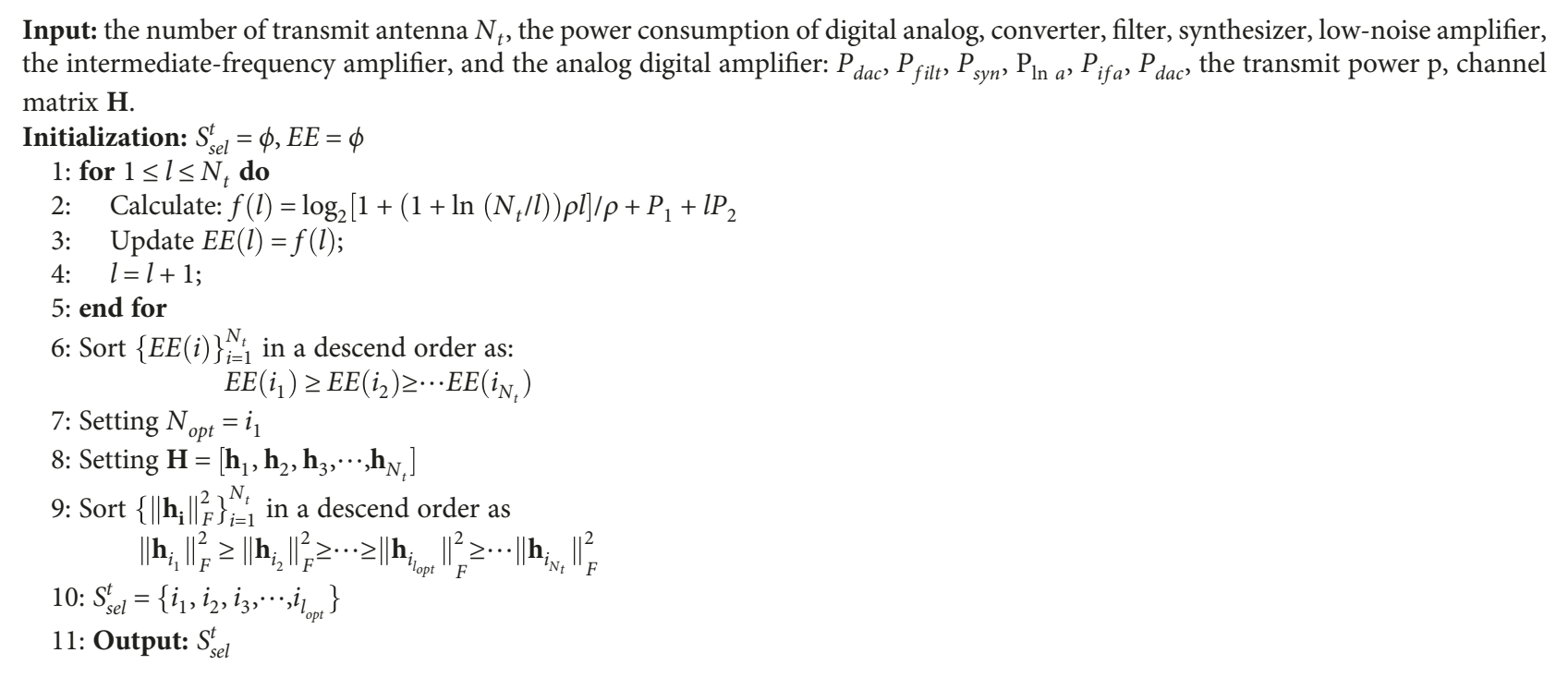

Algorithm 1: The proposed antenna selection scheme.

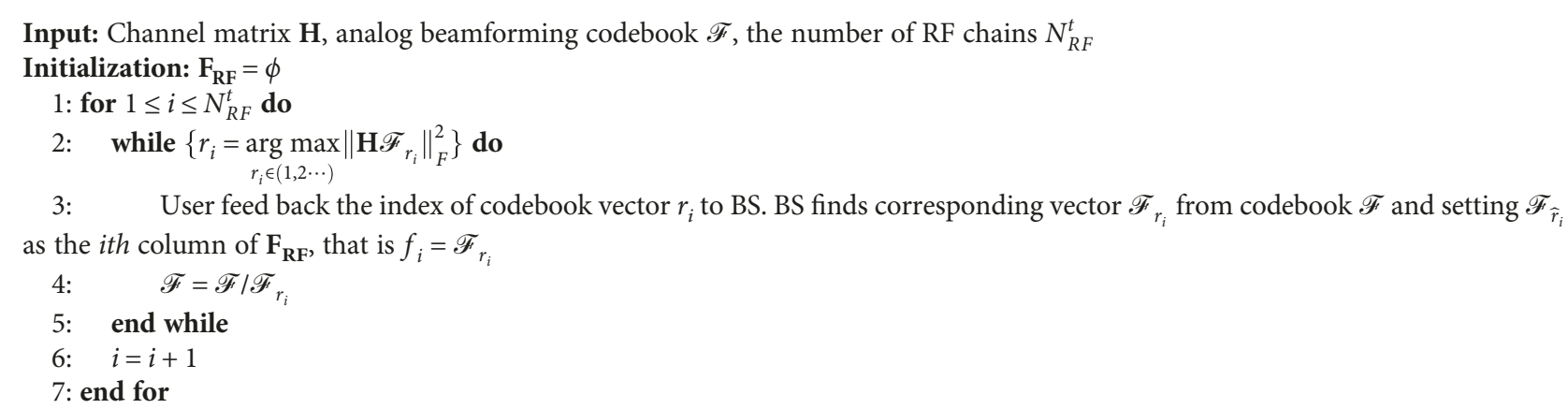

Algorithm 2: Analog precoding scheme.

largest inner product with channel matrix $\mathbf{H}$ and make it as the first column of the analog precoding matrix $\mathbf{F}_{\mathrm{RF}}$.

Then, $i$ is updated by $i+1$, and the procedure above is repeated to find optimal vector $f_{i+1}$ and $f_{i+1}$ is set as the $(i+1)$-th column of analog precoding matrix $\mathbf{F}_{\mathbf{R F}}$.

The procedure is repeated until $i=N_{\mathrm{RF}}^{\mathrm{t}}$. Finally, $\mathrm{F}_{\mathrm{RF}}$ could be obtained, i.e., $\mathbf{F}_{\mathbf{R F}}=\left[f_{1}, f_{2}, f_{3}, \cdots, f_{N_{\mathrm{RF}}^{\mathrm{t}}}\right]$.

6.2. Digital Precoding Design to Improve EE. In this section, we discuss the design of digital beamformer to further improve the EE of the system. After the analog precoding matrix is obtained, the original nonconvex problem is conveyed to a univariate optimization [33] problem which is given by

$$
\begin{aligned}
\mathbf{F}_{\mathbf{B B}} & \underset{\mathbf{F}_{\mathrm{BB}}}{\arg \max }\left(\mathbf{E E}\left(\mathbf{F}_{\mathbf{B B}}\right)\right) \quad \text { s.t. } \quad\left\|\mathbf{F}_{\mathbf{R F}} \mathbf{F}_{\mathbf{B B}}\right\|_{F}^{2} \leq P_{\text {tot }}, \\
\mathbf{E E}\left(\mathbf{F}_{\mathbf{B B}}\right) & =\frac{\log _{2}\left|\mathbf{I}_{N_{\mathrm{t}}}+\mathbf{H}_{\text {sel }}^{\mathbf{t}} \mathbf{F}_{\mathbf{R F}} \mathbf{F}_{\mathbf{B B}} \mathbf{F}_{\mathbf{B B}}^{*} \mathbf{F}_{\mathbf{R F}}^{*}\left(\mathbf{H}_{\text {sel }}^{\mathbf{t}}\right)^{*}\right|}{P_{\mathrm{c}}+\left\|\mathbf{F}_{\mathbf{R F}} \mathbf{F}_{\mathbf{B B}}\right\|_{F}^{2}}
\end{aligned}
$$

There exist many approaches to solve this classical fractional programming problem [34]. At first, we transform it into an equivalent parametric programming problem, $E(\alpha)=0$, where $E(\alpha)$ can be expressed as

$$
E(\alpha)=\max _{\mathbf{F}_{\mathbf{B B}}}\left(R\left(\mathbf{F}_{\mathbf{B B}}\right)-\alpha P\left(\mathbf{F}_{\mathbf{B B}}\right)\right),
$$

$D=\left\{\mathbf{F}_{\mathbf{B B}} \mid\left\|\mathbf{F}_{\mathbf{R F}} \mathbf{F}_{\mathbf{B B}}\right\|_{F}^{2} \leq P_{\text {tot }}\right\}$, where $\alpha$ satisfies $0 \leq \alpha \leq$ $\left(R_{\max } / P_{\mathrm{c}}\right)$ and $R_{\max }=\log \left(1+\left(P_{\text {tot }} / \sigma_{n}^{2}\right)\left\|\mathbf{H}_{\text {sel }}^{\mathbf{t}}\right\|_{F}^{2}\right)$ denotes the max sum rate of the system. The optimal digital precoding matrix $\mathbf{F}_{\mathbf{B B}}^{\mathbf{o p t}}$ which is the solution of (35) is also the optimal solution of (33). The general method to solve (35) is the half-interval search method. It is summarized as in Algorithm 3.

In Algorithm 3, the key step is solving (35). In this section, we will discuss how to solve (35). Problem (35) can be expressed as 


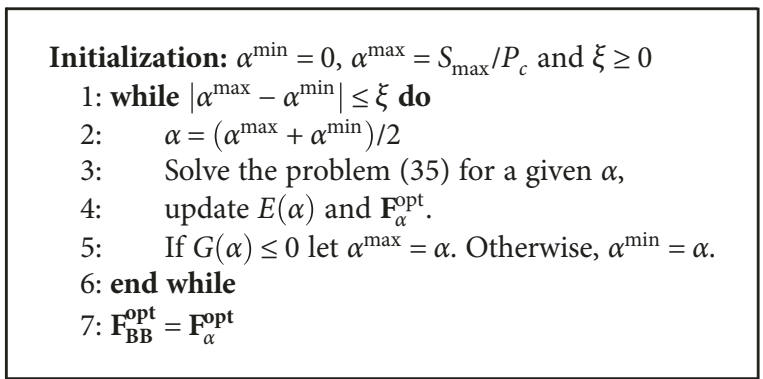

Algorithm 3: Half-interval search method.

$$
\begin{aligned}
\max _{\mathbf{F}} L(\mathbf{F}), \quad \text { s.t. } \quad\|\mathbf{F}\|_{F}^{2} & \leq P_{\text {tot }}, \\
L(\mathbf{F}) & =\log \operatorname{det}\left(\mathbf{I}_{N_{\mathrm{r} x}}+\frac{1}{\sigma_{n}^{2}} \mathbf{H F F}^{H} \mathbf{H}^{H}\right)-\eta \xi\|\mathbf{F}\|_{F}^{2} \\
& =\log \operatorname{det}\left(\mathbf{I}_{N_{\mathrm{tx}}}+\frac{1}{\sigma_{n}^{2}} \mathbf{F F}^{H} \mathbf{H}^{H} \mathbf{H}\right)-\eta \xi\|\mathbf{F}\|_{F}^{2} .
\end{aligned}
$$

According to the knowledge of the determinant, it is obvious that $|\mathbf{I}+\mathbf{M N}|=|\mathbf{I}+\mathbf{N M}|$; (35) could be conveyed into

$$
\begin{aligned}
T(\mathbf{F}) & =\log \left(\mathbf{I}_{N_{H}}+\sigma_{n}^{-2} \sum_{1}^{1 / 2} \mathbf{U}_{1} \mathbf{F F}^{\mathrm{H}} \mathbf{U}_{\mathbf{1}}^{\mathrm{H}} \sum_{1}^{1 / 2}\right)-\alpha \xi\|\mathbf{F}\|^{2} \\
& =\log \left(\mathbf{I}_{\mathbf{N}_{\mathrm{H}}}+\sigma_{n}^{-2} \sum_{1}^{1 / 2}\left(\mathbf{U}_{1}\left(\mathbf{F F}^{\mathbf{H}}\right) \mathbf{U}_{1}^{\mathbf{H}}\right) \sum_{1}^{1 / 2}\right)-\alpha \xi\|\mathbf{F}\|^{2} \\
& =\log \left(\mathbf{I}_{\mathbf{N}_{\mathrm{H}}}+\sigma_{n}^{-2} \sum_{1}^{1 / 2} \mathbf{U}_{1} \mathbf{P} \mathbf{U}_{\mathbf{1}}^{\mathrm{H}} \sum_{1}^{1 / 2}\right)-\alpha \xi\|\mathbf{F}\|^{2} \\
& =\log \left(\mathbf{I}_{\mathbf{N}_{\mathrm{H}}}+\sigma_{n}^{-2} \sum_{1}^{1 / 2} \tilde{\mathbf{P}} \sum_{1}^{1 / 2}\right)-\alpha \xi\|\mathbf{F}\|^{2}
\end{aligned}
$$

where $\mathbf{P}=\mathbf{F} \mathbf{F}^{H}$ and $\tilde{\mathbf{P}}=\mathbf{U}_{\mathbf{1}} \mathbf{P} \mathbf{U}_{\mathbf{1}}^{\mathbf{H}}$; it is obvious that for any matrix $\mathbf{B}$ which is nonnegative, $|\mathbf{B}| \leq \prod \mathbf{B}_{i, i}$. Thus, only if $\mathbf{P}$ is a diagonal matrix will (37) achieve its maximum value. Then, (38) will further expressed as

$$
\begin{aligned}
\max & \left(\log \left[\prod_{j=1}^{N_{H}} 1+p_{j} v_{j} \sigma_{n}^{-2}\right]-\eta \xi \sum_{j=1}^{N_{H}} P_{j}\right) \\
& =\max \sum_{j=1}^{N_{H}} \log \left(1+p_{j} v_{j} \sigma_{n}^{-2}\right)-\eta \xi P_{j} \quad \text { s.t. } \quad \sum_{j=1}^{N_{H}} p_{j} \leq P_{\mathrm{tot}} .
\end{aligned}
$$

The problem in (38) was a convex optimization problem, and its general solution method is to convert it into an unconstrained optimization problem with the Lagrange multiplier method $[35,36]$. Equation (38) could be converted into (39) by the Lagrange multiplier method:

$$
\begin{aligned}
P(\tilde{P}, \lambda) & =-\sum_{i=1}^{N_{H}}\left[\log \left(1+\frac{p_{i} v_{i}}{\sigma_{n}^{2}}\right)-\eta \xi q_{i}\right]+\lambda\left(\sum_{i=1}^{N_{H}} p_{i}-P_{\text {tot }}\right) \\
& =-\sum_{i=1}^{N_{H}}\left[\log \left(1+\frac{v_{i}}{\sigma_{n}^{2}} p_{i}\right)-(\eta+\lambda) p_{i}\right]-\lambda P_{\text {tot }}
\end{aligned}
$$

By solving the KKT conditions of (39), the optimal diagonal entries of $P^{\text {opt }}$ are obtained for a given $\lambda$ via the waterfilling algorithm $[37,38]$.

$$
p_{j}^{\mathrm{opt}}(\lambda)=\left[\frac{1}{\alpha \xi+\lambda}-\frac{\sigma_{n}^{2}}{v_{i}}\right]^{+} \text {. }
$$

$p_{j}^{\text {opt }}$ is a decreasing function of $\lambda$; thus, $\lambda^{o p t}$ could be found by a one-dimensional search algorithm. We proposed a binary water-filling algorithm in this paper. The binary water-filling algorithm is summarized in Algorithm 4.

\section{Simulation Results}

In this section, we present simulation results to evaluate the performance of the proposed algorithm in terms of $\mathbf{E E}$ and the sum rate. Specially, the simulation parameters are summarized in Table 1. In this section, we first compare the performance of different algorithms in terms of $\mathbf{E E}$ with different antenna array sizes. Then, we compare the performance of the proposed algorithm with a conventional algorithm under different power constraints in terms of EE. At last, we compare the performance of different precoding schemes in terms of the sum rate.

Figure 2 compares the performance between the energyefficient algorithm and spectral efficient algorithm under different SNRs in terms of SE. As Figure 2 shows, with the increase in SNR, the system performance of different algorithms increases, but the performance of the pure digital precoding system is optimal. The performance of the spectral efficient hybrid precoding scheme is close to that of the digital precoding scheme, and the performance of the energyefficient algorithm is lower than that of the spectral efficient algorithm in terms of SE. This is because the energyefficient algorithm sacrifices part of the spectrum efficiency to improve the energy efficiency of the system. And, for the same algorithm (energy-efficient algorithm/spectral efficient algorithm), using a different number of RFs will lead to a different performance in terms of spectral efficiency. As can be seen from the figure, under the same SNR condition, the higher the number of radio frequency chains, the better the system performance.

Figure 3 compares the EE performance between the energy-efficient algorithm and spectral efficient algorithm under different transmit power constraints. As Figure 3 shows, at low power constraint, the energy-efficient performance of the proposed algorithm is exactly close to that of the spectral efficient algorithm. As the transmit power increases, the EE of the proposed algorithm remains a constant but shows a decline in the spectral efficient algorithm. 


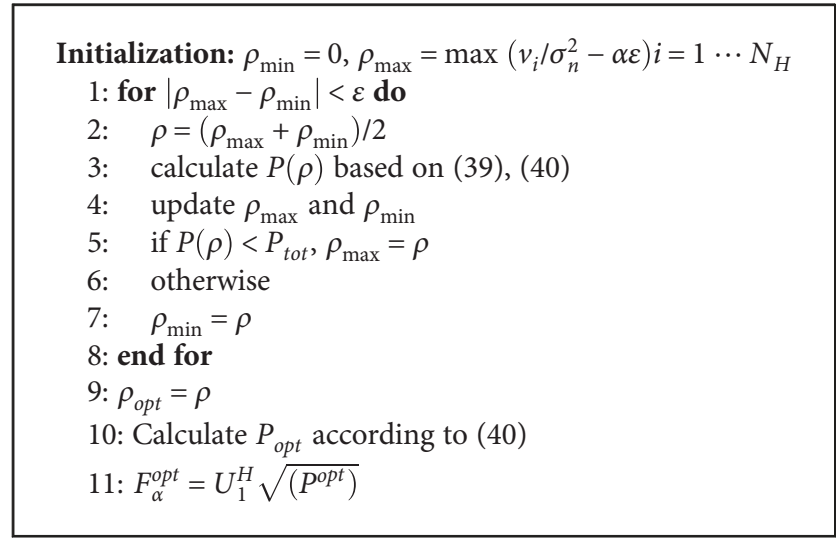

Algorithm 4: Binary iterative water-filling algorithm.

TABLE 1: Simulation parameters.

\begin{tabular}{lc}
\hline Parameters & Value \\
\hline Number of antennas & $0 \sim 180$ \\
Channel model & IEEE 802.11ad living room \\
Number of users & 1 \\
Carrier frequency $(\mathrm{GHz})$ & 60 \\
Bandwidth & 2.56 \\
Power amplifier efficiency $\rho(\%)$ & 26 \\
Modulation coding scheme & 16PSK, $1 / 2$ turbo codes \\
Simulation frames & 20000 \\
Number of RF chains & $2 \sim 20$ \\
\hline
\end{tabular}

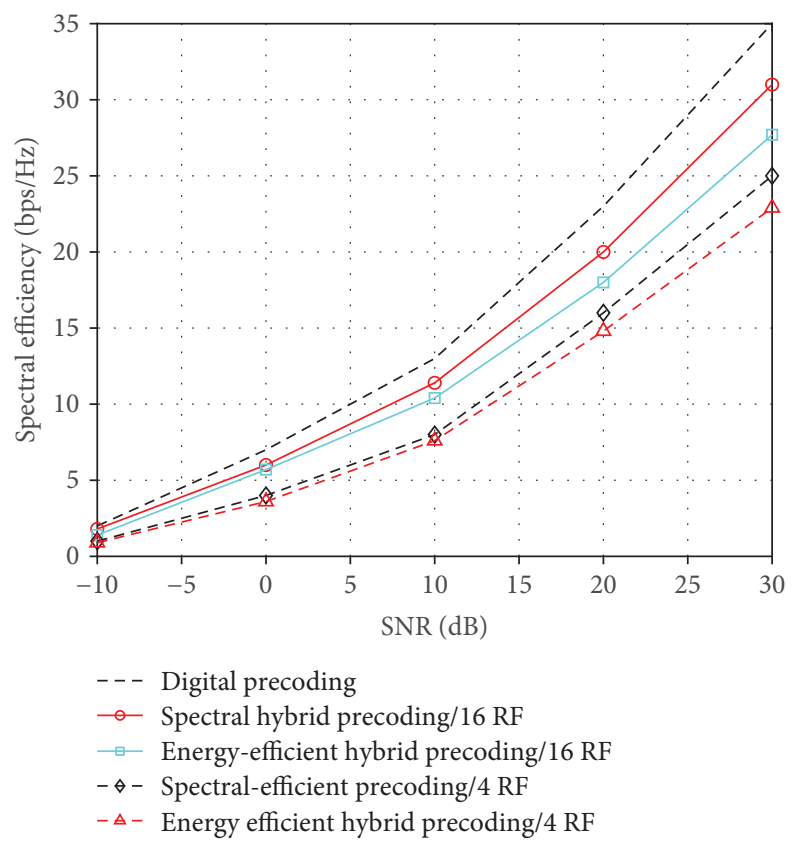

FIGURE 2: SE comparison of different precoding algorithms under different SNRs.

The reason is that in the spectral efficient algorithm, the increase in the sum rate cannot keep path with the increase in power consumption.

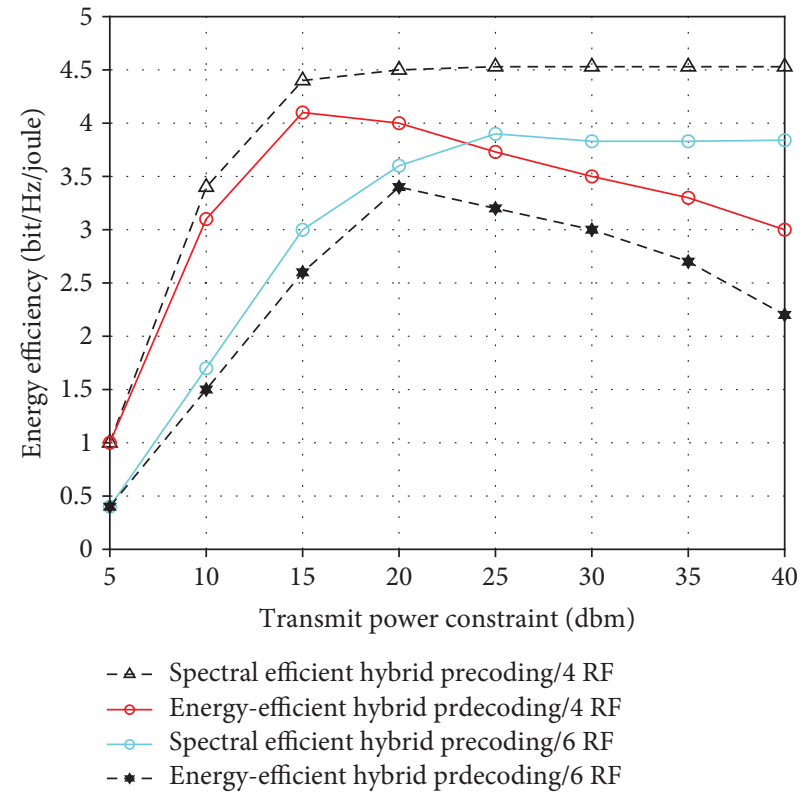

FIGURE 3: EE comparison of different precoding algorithms under different power constraints.

Figure 4 compares the performance between the energyefficient algorithm and spectral efficient algorithm under different antenna array sizes in terms of EE. As Figure 4 shows, there exists an optimal antenna array size to maximize the $\mathbf{E E}$ of the system.

The EE increases at first and then decreases as the number of transmit antennas increases. The more power constraint there is, the larger the antenna array size is. It is reasonable, because once the number of transmit antennas $N_{\mathrm{t}}$ increases over a certain value, the increase in EE cannot compensate for the negative influence of power consumption increase $P_{\mathrm{c}}$.

Figure 5 compares the energy efficiency achieved under a given optimal number of antennas $N_{\text {opt }}$ and transmits power but a different number of RF chains. As Figure 5 shows, under the condition that the optimal number of transmitting antennas $N_{\text {opt }}$ is 80 , with the increase in the number of RF chains, the $\mathbf{E E}$ of the system increases first and then decreases. It can be seen from the figure that the optimum RF number is 8 . Meanwhile, it can be seen from Figure 5 that when the number of transmitting antennas is the same, the optimal number of the RF chain is the same. At this time, the larger the transmitting power of the system, the higher the EE that the system can achieve.

Figure 6 compares the SE achieved under a given optimal number of antennas $N_{\text {opt }}$ and transmit power but a different number of RF chains. As Figure 5 shows, when the optimal number of transmitting antennas is 80 , the $\mathbf{E E}$ of the system increases at first and then is kept constant with the increase in the number of radio frequency chains. As can be seen from the figure, when the number of RF chains increases from 1 to 10 under the condition that $N_{\mathrm{t}}=80$ and $p=30 \mathrm{dbm}$, the RF of the system increases. When the 


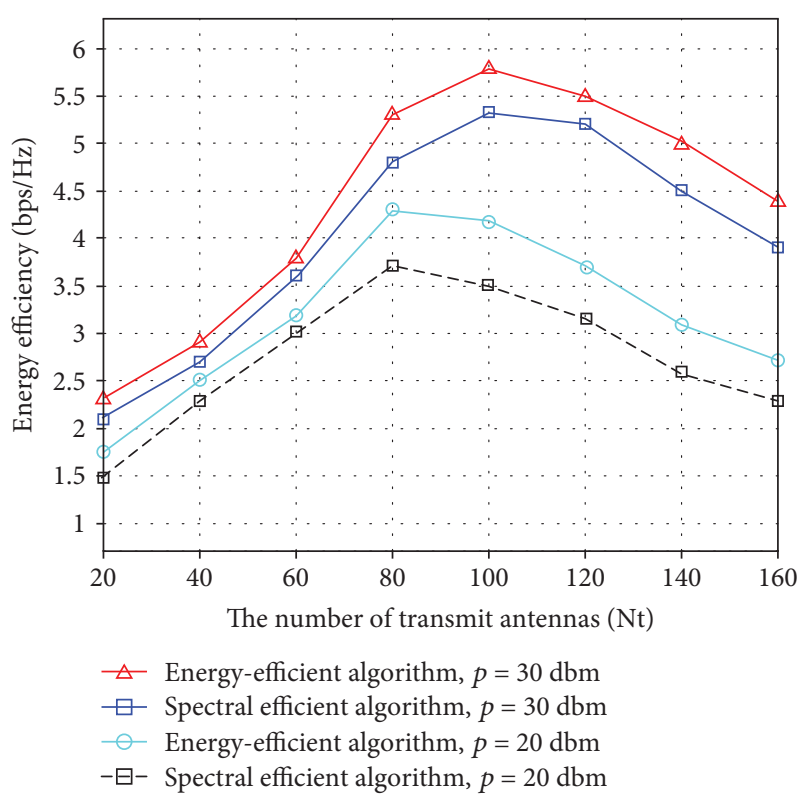

FIGURE 4: EE comparison of different precoding algorithms under different transmit antennas.

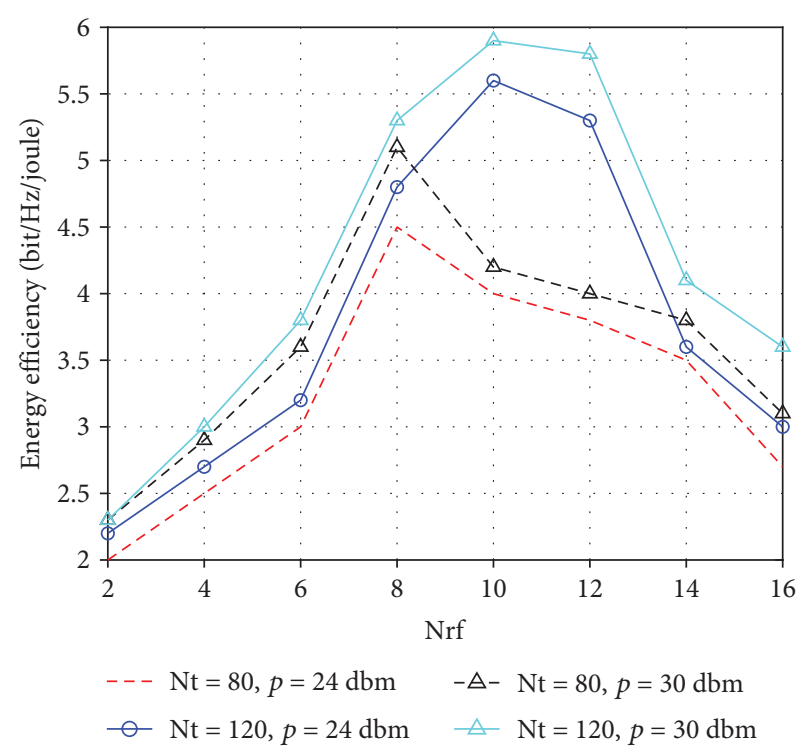

FIGURE 5: EE comparison of the energy-efficient precoding scheme under different RF chains.

number of RF chains is greater than 10 , the SE of the system will be constant. However, with the increase in the number of RF, the power consumption of the system will increase, which will lead to the decrease in the $\mathbf{E E}$ of the system. This is consistent with the result of Figure 6. In Figure 6, we can conclude that the EE of the system reaches its maximum when the number of $\mathbf{R F}$ chains is 8 under the condition that $p=30 \mathrm{dbm}$ and $N_{t}=80$. Meanwhile, the larger the number of transmitting antennas $N_{\mathrm{t}}$, the greater the maximum SE the system can achieve.

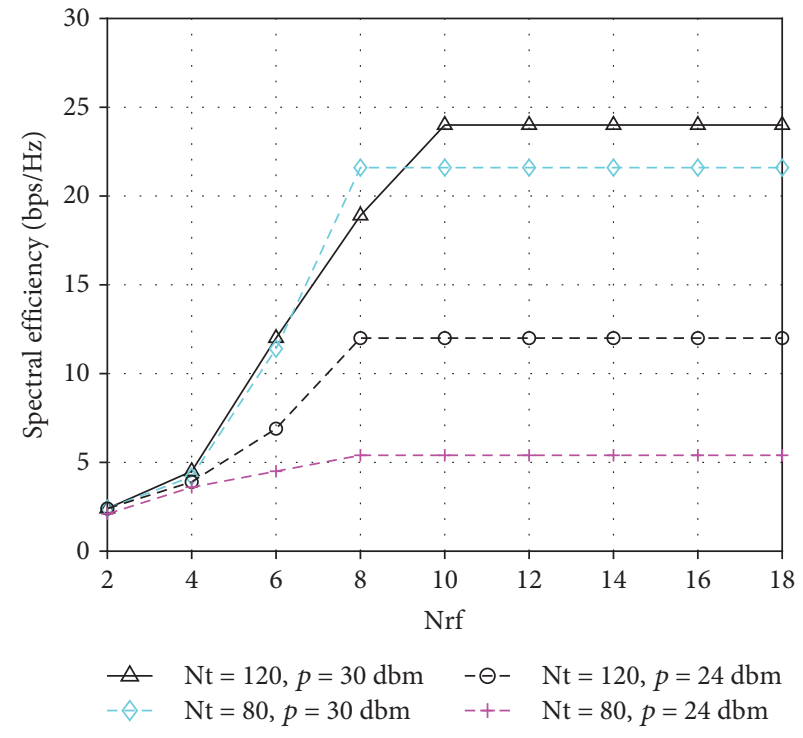

FIgURE 6: SE comparison of the energy-efficient precoding scheme under different radio frequency chains.

\section{Conclusions}

In this paper, an energy-efficient hybrid precoding scheme for single-user mmWave massive MIMO systems was considered. First, we calculate the optimal number of transmit antennas according to the channel state information and then design an antenna selection algorithm to select the antenna to maximize the channel gain. Finally, the EE of the system is optimized by digital precoding. Numerical results show that the near-optimal hybrid precoding algorithm achieved a near-optimal EE performance. Simulation results show that the algorithm we proposed can achieve higher EE than the traditional precoding scheme can. In future work, we will study on the joint optimization of transmit antennas, receive antennas, and precoder to improve the EE of the system.

\section{Data Availability}

The data used to support the findings of this study are available from the corresponding author upon request.

\section{Conflicts of Interest}

The authors declare that they have no conflicts of interest.

\section{Acknowledgments}

This paper is sponsored by the National Natural Science Foundation of China under Grant 61871321, National Science and Technology Major Project under Grant 2016 ZX03001016, and Innovation Team Project of Shaanxi Province under Grant 2017KCT-30-02. 


\section{References}

[1] D. W. Bliss, A. M. Chan, and N. B. Chang, "Mimo wireless communication channel phenomenology," IEEE Transactions on Antennas and Propagation, vol. 52, no. 8, pp. 2073-2082, 2004.

[2] K.-K. Wong, R. D. Murch, and K. B. Letaief, "Performance enhancement of multiuser mimo wireless communication systems," IEEE Transactions on Communications, vol. 50, no. 12, pp. 1960-1970, 2002.

[3] T. S. Rappaport, G. R. MacCartney, M. K. Samimi, and S. Sun, "Wide-band millimeter-wave propagation measurements and channel models for future wireless communication system design," IEEE Transactions on Communications, vol. 63, no. 9, pp. 3029-3056, 2015.

[4] C. Rusu, R. Mendez-Rial, N. Gonzalez-Prelcic, and R. W. Heath, "Low complexity hybrid precoding strategies for millimeter wave communication systems," IEEE Transactions on Wireless Communications, vol. 15, no. 12, pp. 8380-8393, 2016.

[5] T. E. Bogale and L. B. le, "Massive mimo and mmwave for $5 \mathrm{~g}$ wireless hetnet: potential benefits and challenges," IEEE Vehicular Technology Magazine, vol. 11, no. 1, pp. 64-75, 2016.

[6] T. Halinen, A. A. Dowhuszko, and J. Hamalainen, "Performance of distributed beamforming for dense relay deployments in the presence of limited feedback information," EURASIP Journal on Advances in Signal Processing, vol. 2013, no. 1, 2013.

[7] J. Zhang, X. Huang, V. Dyadyuk, and Y. Guo, "Massive hybrid antenna array for millimeter-wave cellular communications," IEEE Wireless Communications, vol. 22, no. 1, pp. 79-87, 2015.

[8] J. Singh and S. Ramakrishna, "On the feasibility of beamforming in millimeter wave communication systems with multiple antenna arrays," IEEE Transactions on Wireless Communications, vol. 14, no. 5, pp. 3802-3808, 2014.

[9] X. Gao, L. Dai, S. Han, C. L. I, and R. W. Heath, "Energyefficient hybrid analog and digital precoding for mmwave mimo systems with large antenna arrays," IEEE Journal on Selected Areas in Communications, vol. 34, no. 4, pp. 9981009, 2016.

[10] D. W. Browne, M. Manteghi, M. P. Fitz, and Y. Rahmat-Samii, "Experiments with compact antenna arrays for mimo radio communications," IEEE Transactions on Antennas and Propagation, vol. 54, no. 11, pp. 3239-3250, 2006.

[11] A. M. Sayeed and V. Raghavan, "Maximizing mimo capacity in sparse multipath with reconfigurable antenna arrays," IEEE Journal of Selected Topics in Signal Processing, vol. 1, no. 1, pp. 156-166, 2007.

[12] Z. Pi and F. Khan, "An introduction to millimeter-wave mobile broad-band systems," IEEE Communications Magazine, vol. 49, no. 6, pp. 101-107, 2011.

[13] X. Zhu, Z. Wang, L. Dai, and Q. Wang, “Adaptive hybrid precoding for multi-user massive mimo," IEEE Communications Letters, vol. 20, no. 4, pp. 776-779, 2016.

[14] P. Zhao and Z. Wang, "Hybrid precoding for millimeter wave communications with fully connected subarrays," IEEE Communications Letters, vol. 22, no. 10, pp. 2160-2163, 2018.

[15] C. C. Hu and J. H. Zhang, "Hybrid precoding design for adaptive subconnected structures in millimeter-wave mimo systems," IEEE Systems Journal, vol. 13, no. 1, pp. 137-146, 2019.
[16] T. Huang, M. Linhua, H. Xing, H. Shaocheng, S. Kangning, and L. Shiping, "Practical hybrid precoding algorithm for millimeter wave massive MIMO," Journal of Electronics \& Information Technology, vol. 39, no. 8, pp. 1788-1795, 2017.

[17] M. Majidzadeh, A. Moilanen, N. Tervo, H. Pennanen, A. Tolli, and M. Latva-Aho, "Hybrid beamforming for single-user mimo with partially connected RF architecture," in 2017 European Conference on Networks and Communications (EuCNC), Oulu, Finland, 2017.

[18] M. Hanif, H. C. Yang, G. Boudreau, E. Sich, and H. Seyedmehdi, "Practical hybrid precoding for multi-user massive mimo systems," in 2016 IEEE Canadian Conference on Electrical and Computer Engineering (CCECE), Vancouver, BC, Canada, 2016.

[19] N. Li, Z. Wei, H. Yang et al., "Hybrid Precoding for mmWave Massive MIMO Systems With Partially Connected Structure," IEEE Access, vol. 5, no. 1, pp. 1514215151, 2017.

[20] S. Park, A. Alkhateeb, and R. W. Heath, "Dynamic subarrays for hybrid precoding in wideband mmWave MIMO systems," IEEE Transactions on Wireless Communications, vol. 16, no. 5, pp. 2907-2920, 2017.

[21] Y. Kai and B. E. Ottersten, "Models for MIMO propagation channels: a review," Wireless Communications and Mobile Computing, vol. 2, no. 7, 666 pages, 2002.

[22] F. Heliot, M. A. Imran, and R. Tafazolli, "On the energy efficiency gain of mimo communication under various power consumption models," in Future Network \& Mobile Summit, Warsaw, Poland, 2011.

[23] K. Wang, T. Nirmalathas, C. Lim, and S. Skafidas, "Power-consumption comparison of high-speed indoor millimeter-wave $60 \mathrm{GHz}$ communication system and optical wireless systems," in Asia Pactific Microwave Photontics Conference in Japan, Kyoto, 2012.

[24] C. Ma, C. Pan, Y. Pan, X. Han, and C. Ming, "Energy-efficient hybrid precoding for millimeter wave MIMO systems," in International Conference on Communications \& Networking in China, Shanghai, China, 2016.

[25] H. Li, L. Song, and M. Debbah, "Energy efficiency of large-scale multiple antenna systems with transmit antenna selection," IEEE Transactions on Communications, vol. 62, no. 2, pp. 638-647, 2014.

[26] A. Moudafi, "A proximal iterative approach to a nonconvex optimization problem," Nonlinear Analysis: Theory, Methods \& Applications, vol. 72, no. 2, pp. 704-709, 2010.

[27] X. Y. Wang, X. W. Jiang, and J. X. Dai, "Homotopy method for nonconvex optimization problem," Journal of Jilin University, vol. 52, no. 2, pp. 273-276, 2014.

[28] Y. Bian, B. Mirzasoleiman, J. M. Buhmann, and A. Krause, "Guaranteed non-convex optimization: submodular maximization over continuous domains," 2016, arXiv: 1606.05615.

[29] P. Jain and P. Kar, "Non-convex optimization for machine learning," Foundations and Trends ${ }^{\circledR}$ in Machine Learning, vol. 10, no. 3-4, pp. 142-336, 2017.

[30] J. Jing, C. Xiaoxue, and X. Yongbin, "Energy-efficiency based downlink multi-user hybrid beamforming for millimeter wave massive mimo system," The Journal of China Universities of Posts and Telecommunications, vol. 23, no. 4, pp. 53-62, 2016. 
[31] J. Jing, K. Deting, L. Guangyue, W. Junxuan, and Y. Wujun, "User scheduling algorithm for mmwave FDMA massive MU-MIMO system with hybrid beamforming," in Proceedings of the 8th International Conference on Signal Processing Systems - ICSPS 2016, pp. 194-198, Auckland, New Zealand, 2016.

[32] T. E. Bogale and L. B. Le, "Pilot optimization and channel estimation for multiuser massive mimo systems," in 2014 48th Annual Conference on Information Sciences and Systems (CISS), Princeton, NJ, USA, 2014.

[33] A. Zilinskas and G. Gimbutiene, "On one-step worst-case optimal trisection in univariate bi-objective Lipschitz optimization," Communications in Nonlinear Science and Numerical Simulation, vol. 35, pp. 123-136, 2016.

[34] J. P. Crouzeix and J. A. Ferland, "Algorithms for generalized fractional programming," Mathematical Programming, vol. 52, no. 1-3, pp. 191-207, 1991.

[35] D. P. Bertsekas, Constrained Optimization and Lagrange Multiplier Methods, Academic Press, 1982.

[36] G. Haeser and M. L. Schuverdt, "On approximate KKT condition and its extension to continuous variational inequalities," Journal of Optimization Theory and Applications, vol. 149, no. 3, pp. 528-539, 2011.

[37] M. Kobayashi and G. Caire, "An iterative water-filling algorithm for maximum weighted sum-rate of Gaussian MIMOBC," IEEE Journal on Selected Areas in Communications, vol. 24, no. 8, pp. 1640-1646, 2006.

[38] R. S. Prabhu and B. Daneshrad, "An energy-efficient waterfilling algorithm for OFDM systems," in 2010 IEEE International Conference on Communications, Cape Town, South Africa, 2010. 


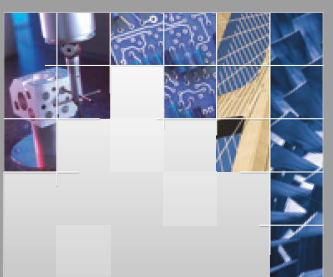

\section{Enfincering}
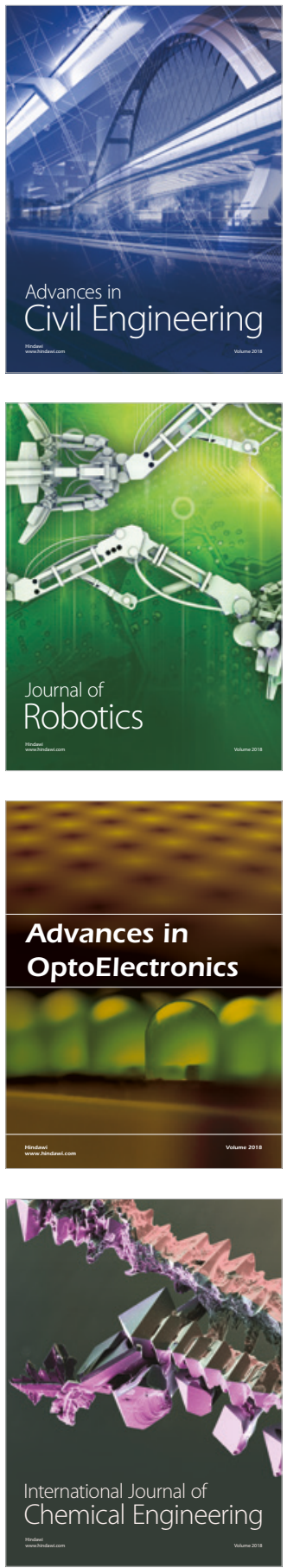

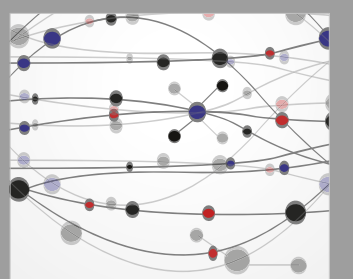

\section{Rotating \\ Machinery}

The Scientific World Journal

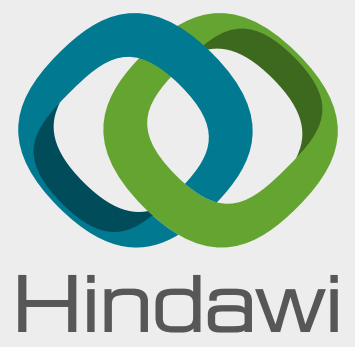

Submit your manuscripts at

www.hindawi.com
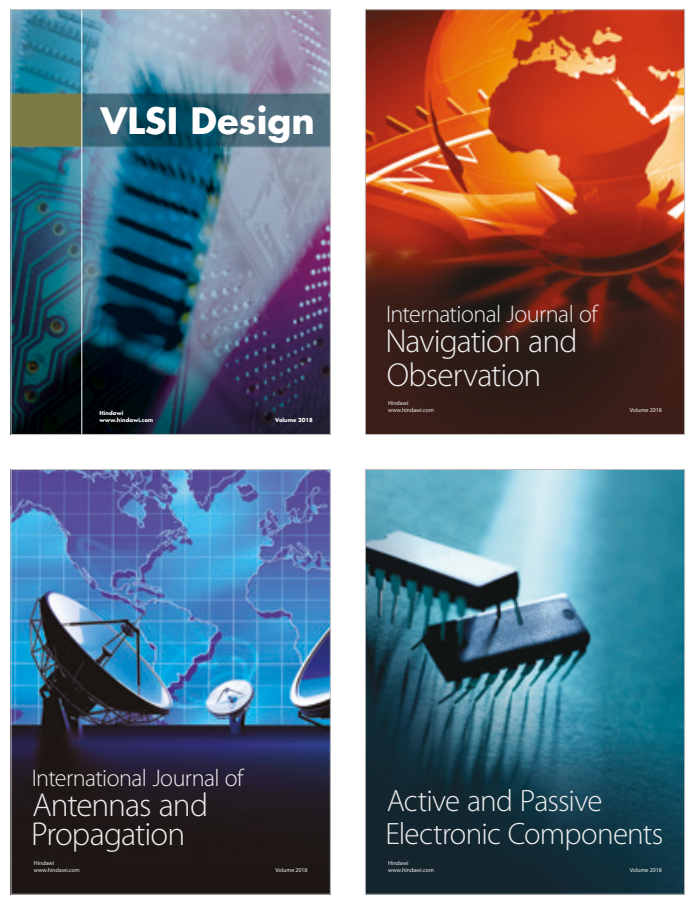
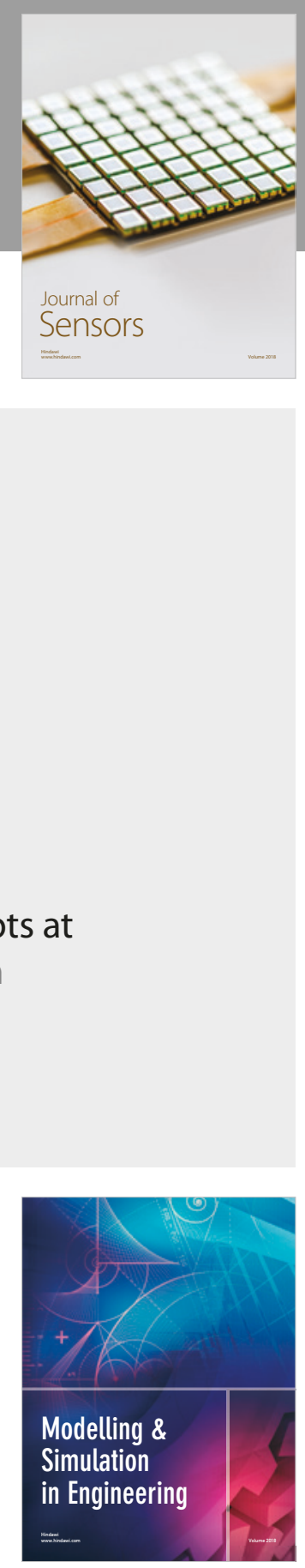

\section{Advances \\ Multimedia}
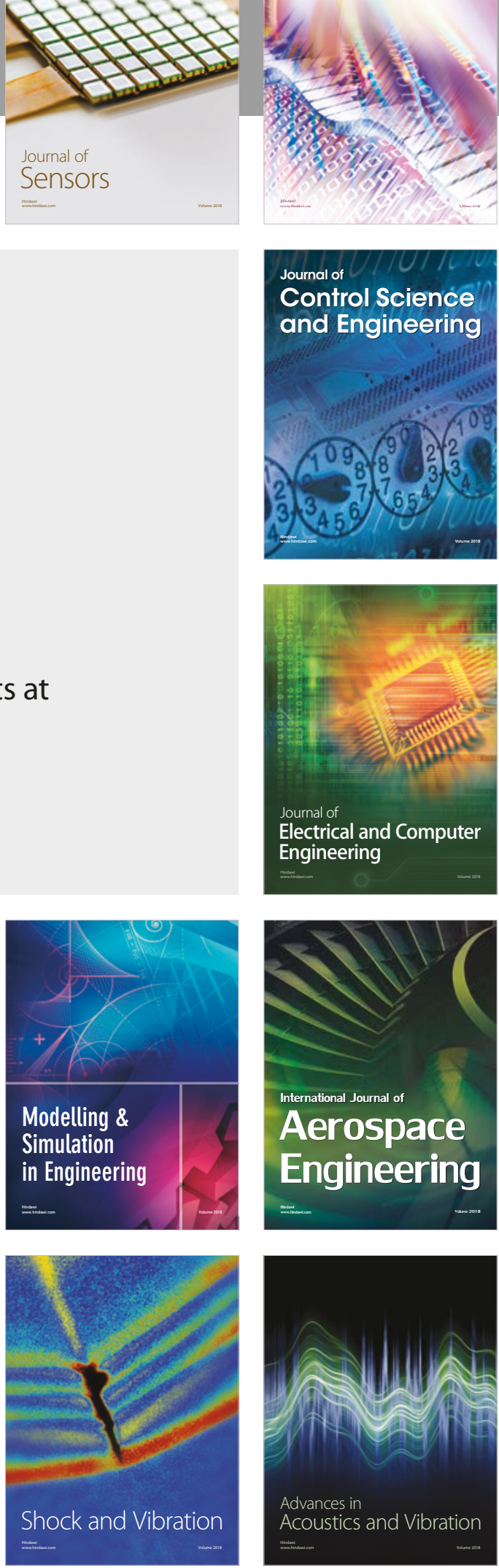